\title{
Temperature variation of the pseudogap in underdoped cuprates
}

\author{
Andrey V. Chubukov ${ }^{1}$ and Jörg Schmalian ${ }^{2}$ \\ 1 Department of Physics, University of Wisconsin, Madison, WI 53706 \\ 2 Department of Physics, University of Illinois at Urbana-Champaign, Urbana, IL 61801
}

(October 20, 1997)

\begin{abstract}
We consider thermal evolution of the spectral function $A(\Omega, T)$ in underdoped cuprates at $T>T_{\mathrm{c}}$. We find that in the strong coupling limit, the fermionic Green's function near $(0, \pi)$ behaves as $G^{-1}(\Omega) \propto \sqrt{\Omega}$ in the whole frequency range relevant to photoemission experiments. The analysis of the pairing problem with this form of $G$ yields a leading edge gap in $A(\Omega)$ and a broad maximum at larger frequencies. We find that classical fluctuations predominantly add a frequency independent term to $G^{-1}(\Omega)$. This term destroys the leading edge gap at $T=T^{*}(\mathbf{k})$ which has a maximum value of about $200 K$ near $(0, \pi)$.
\end{abstract}

The pseudogap phenomenon in the normal state properties of underdoped cuprates is one of the key issues in the field of high- $T_{\mathrm{c}}$ superconductivity. NMR, transport, optical and photoemission measurements [1] all demonstrate that underdoped cuprates behave in many respects like a $d$-wave superconductor at temperatures which can be several times larger than actual $T_{\mathrm{c}}$. In photoemission measurements, the evidence for a pseudogap is based on the observation of a leading edge gap (LEG) in the quasiparticle spectral function $A(\Omega)$ near $(0, \pi)$ and symmetry related points [2,3]. Contrary to situation below $T_{\mathrm{c}}$, however, the LEG is not accompanied by a sharp quasiparticle peak above it. Instead, the spectral function is flat above the LEG and only displays a broad maximum at much larger frequencies [2]. This form of $A(\Omega)$ exists between $T_{\mathrm{c}}$, which can be very small in strongly underdoped cuprates, and some higher temperature, $T^{*}(k)$ which is momentum dependent [3] and has a maximum value of about $200 K$ near $(0, \pi)$. Similar observations have been made recently in tunneling experiments [4.

In a recent paper [5], one of us argued that the LEG naturally appears in the in the "magnetic" scenario for cuprates due to strong quantum magnetic fluctuations which give rise to $d$-wave precursors above $T_{\mathrm{c}}$, but simultaneously almost destroy the quasiparticle peak near $(0, \pi)$. These two effects yield a LEG without a quasiparticle peak, in agreement with the data.

In this paper we show that, as temperature increases, magnetic fluctuations loose their dynamics and progressively acquire a classical, quasistatic behavior. We show that this effect acts against $d$-wave precursors. We further show that the LEG is filled in by excitations at temperatures which are comparable to a typical spinfluctuation frequency and also depend on the momentum, being largest near $(0, \pi)$.

As in earlier studies [5]7], our consideration is based on the spin-fermion model in which itinerant fermions which form a Fermi liquid with a large, Luttinger-type Fermi surface interact with their collective spin degrees of freedom by

$$
\mathcal{H}_{\mathrm{s}-\mathrm{f}}=g \sum_{\mathbf{k q}, \gamma \delta} c_{\mathbf{k}, \gamma}^{\dagger} \vec{\sigma}_{\gamma, \delta} c_{\mathbf{k}+\mathbf{q}, \delta} \vec{S}_{-\mathbf{q}} .
$$

Here $g$ is the coupling constant which is assumed to increase as the system approaches half-filling, $\sigma_{i}$ are the Pauli matrices, and $\vec{S}_{\mathbf{q}}$ are collective spin variables which are characterized by their full spin susceptibility $\chi_{\gamma \delta}(q, \omega)=\delta_{\gamma \delta} \tilde{\chi} /\left(1+(\tilde{\mathbf{q}} \xi)^{2}-i \omega / \omega_{\text {sf }}-\omega^{2} / \Delta^{2}\right)$. Here $\xi$ is the correlation length, $\Delta \propto \xi^{-1}$, and $\tilde{\mathbf{q}}=\mathbf{Q}-\mathbf{q}$ where $\mathbf{Q}$ is the momentum at which the static susceptibility is peaked. At small doping, $\mathbf{Q}$ is either equal or very close to the antiferromagnetic momentum $(\pi, \pi)$. Further, $\tilde{\chi}=a \xi^{2}$ is the static susceptibility at $\mathbf{q}=\mathbf{Q}$ and $\omega_{\text {sf }}$ is the typical frequency of overdamped spin fluctuations. A fit to NMR experiments yields $a \sim 25$ states $/ \mathrm{eV}$, $\omega_{\text {sf }} \sim 10 \mathrm{meV}$ and $\xi \sim 3-4$ at the onset temperature for a LEG [8]. The value of $\Delta$ is unknown, but since $\omega^{2}$ term in $\chi$ accounts for spin waves, it is reasonable to estimate $\Delta \sim v_{s} \xi^{-1}$ where $v_{s}$ is the spin-wave velocity.

Our goal is to obtain the form of the quasiparticle spectral function $A(\mathbf{k}, \Omega)=(1 / \pi)|\operatorname{Im} G(\mathbf{k}, \Omega)|$ where $G^{-1}(\mathbf{k}, \Omega)=G_{0}^{-1}(\mathbf{k}, \Omega)-\Sigma(\mathbf{k}, \Omega)$. This can be most straightforwardly done by first evaluating $\Sigma\left(\mathbf{k}, \Omega_{m}\right)$ for discrete Matsubara frequencies $\Omega_{m}=\pi T(2 m+1)$, and then performing analytical continuation to real frequencies. To avoid unnecessary complications, we also restrict our consideration to the points in momentum space $\mathbf{k}=\mathbf{k}_{\mathrm{hs}}$ where $\epsilon_{\mathbf{k}}=\epsilon_{\mathbf{k}+\mathbf{Q}}=\mu$, and the self-energy has the largest value. In cuprates these points (hot spots) are located near $(0, \pi)$ and symmetry related points. Finally, we restrict our consideration to second-order term in $g$ - previous studies indicate that the second-order result for the fermionic self-energy is dominant even for large values of the coupling constant [6].

To second order in $g, \Sigma\left(\mathbf{k}, \Omega_{m}\right)$ is given by

$$
\Sigma\left(\mathbf{k}, \Omega_{m}\right)=3 g^{2} T \sum_{\mathbf{q} n} G_{0}\left(\mathbf{k}+\mathbf{q}, \Omega_{m}+\omega_{n}\right) \chi_{\gamma \gamma}\left(\mathbf{q}, \omega_{n}\right)
$$

where $\omega_{n}=2 \pi n T$ and $G_{0}$ is a bare fermionic Green's function which near the Fermi surface has a form $G_{0}\left(\mathbf{k}, \omega_{n}\right)=Z_{0} /\left(i \omega_{n}-\epsilon_{\mathbf{k}}\right)$. Expanding in $\epsilon_{\mathbf{k}+\mathbf{q}}$ to linear order in a deviation from a hot spot and performing integration over momentum we obtain

$$
\Sigma\left(\mathbf{k}_{\mathrm{hs}}, \Omega_{m}\right)=-2 i T\left(\frac{g}{g_{0}}\right)^{2} \times
$$




$$
\sum_{n} \frac{\Omega_{m}+\omega_{n}}{\left|\Omega_{m}+\omega_{n}\right|} K_{n} \cot ^{-1}\left(\frac{K_{n}\left|\Omega_{m}+\omega_{n}\right|}{v \xi^{-1}}\right)
$$

where $K_{n}^{-2}=1+\left|\omega_{n}\right| / \omega_{\mathrm{sf}}+\omega_{n}^{2} / \Delta^{2}-\left(\left(\Omega_{m}+\omega_{n}\right) /\left(v \xi^{-1}\right)\right)^{2}$, and we introduced $g_{0}^{2}=4 \pi v \xi^{-1} /\left(3 a Z_{0}^{2}\right)$ where $v$ is the Fermi velocity at a hot spot. The fits to photoemission and NMR data above $T^{*}$ yield, respectively, $v \sim 0.4 \mathrm{eV}$ and $v \xi^{-1} / \omega_{\mathrm{sf}} \sim 10$ independent on temperature. For definiteness, we will also choose $v \xi^{-1} / \Delta=1$. We have done calculations for larger ratios (2 and 4) and obtain little difference in the results.

The summation over $n$ can be performed explicitly for $\Omega_{m} \ll v \xi^{-1}$. For these $\Omega_{m}$, the use of the Poisson summation formula yields

$\Sigma\left(\mathbf{k}_{\mathrm{hs}}, \Omega_{m}\right)=-i\left(\frac{g}{g_{0}}\right)^{2}\left(\frac{2 \Omega_{m}}{1+\sqrt{1+\frac{\left|\Omega_{m}\right|}{\omega_{\mathrm{sf}}}}}+\Lambda\left(\Omega_{m}, T\right)\right)$

where $\Lambda\left(\Omega_{m}, T\right)$ is expressed in terms of Fresnel integrals. The first term in this formula is a quantum result while the second term accounts for thermal effects. At low $T \leq$ $\omega_{\text {sf }}$, the use of the asymptotic form of Fresnel integrals yields

$$
\Lambda\left(\Omega_{m}, T\right)=\operatorname{sign} \Omega_{m} \frac{(\pi T)^{2}}{4 \omega_{\mathrm{sf}}} f\left(\frac{\Omega_{m}}{\omega_{\mathrm{sf}}}\right),
$$

where $f(0)=1-0.32\left(\pi T / \omega_{\mathrm{sf}}\right)^{2}+\ldots$ and $f(x \gg 1)=$ $(2 / 3)\left(1-0.28\left(\pi T / \omega_{\mathrm{sf}}\right)^{2}+\ldots\right.$. The leading term in the expansion obviously yields a conventional Fermi-liquid result for the self-energy. We see, however, that the typical scale above which Fermi-liquid expansion breaks down for both external frequency and temperature is given by $\omega_{\text {sf }}$ which is rather small in cuprates. In the opposite limit of "high" $T, \Omega \gg \omega_{\text {sf }}$ (but still, $T, \Omega \ll v \xi^{-1}$ ), the self-energy has the form

$$
\Sigma\left(\mathbf{k}_{\mathrm{hs}}, \Omega\right)=-2\left(\frac{g}{g_{0}}\right)^{2}\left(\Omega \omega_{\mathrm{sf}}\right)^{1 / 2}(1+i \alpha(\Omega)),
$$

where $\alpha(\Omega)=1+\beta \sqrt{\omega_{\mathrm{sf}} / \Omega}$, and $\beta=\pi T /\left(2 \omega_{\mathrm{sf}}\right)$. In this limit, the spin fluctuations are essentially static, the thermal part of $\Sigma\left(\mathbf{k}_{\mathrm{hs}}, \Omega\right)$ dominates, and the self-energy is linear in $T$. We however studied the crossover between quadratic and linear in $T$ behavior of $\beta$ in more detail and found that although the deviations from the Fermiliquid behavior begin at rather small $T$, the asymptotic, linear in $T$ form of $\beta$ is recovered only at $T \gg \omega_{\mathrm{sf}}$, i.e, the crossover region is numerically rather wide.

We will show below that the onset temperature for the LEG falls into the intermediate temperature range where quantum and classical contributions to the selfenergy are of about the same strength. But before, we briefly review the earlier results [5] of a purely quantum consideration when $\beta=0$ which was argued to give rise to a LEG without a quasiparticle peak. The key points of the quantum consideration are:

(i) The self-energy as in (4) survives in some region around a hot spot with the width in $\mathbf{k}$-space $\sim \Omega / \omega_{\mathrm{sf}} \xi^{2}$. Numerical estimates show that for $\Omega \geq \omega_{\text {sf }}$, this region is rather wide and, e.g., for $\mathbf{Q}=(\pi, \pi)$ it includes $(0, \pi)$ and symmetry related points.

(ii) When $g$ becomes few times larger than $g_{0}$ (which is likely to happen when the system approaches optimal doping from the overdoped side), the self-energy overshadows the bare fermionic dispersion such that $G^{-1}(\mathbf{k}, \Omega) \approx \Sigma\left(\mathbf{k}_{\mathrm{hs}}, \Omega\right)$. As real and imaginary parts of $\Sigma\left(\mathbf{k}_{\mathrm{hs}}, \Omega\right)$ in (4) are the same for $\beta=0$, the dominance of the self-energy over bare dispersion leads to a transform of a spectral weight to higher frequencies, and to a near-destruction of the quasiparticle peak near hot spots.

(iii) The analysis of the pairing susceptibility shows that though the $d$-wave pairing susceptibility turns out to be substantially enhanced for $g>g_{0}$, the neardestruction of the quasiparticle peak prevents the system to become an actual superconductor down to very small $T$. At the same time, due to the enhancement and narrowing of the pairing susceptibility at $g>g_{0}$, the frequency shift which one obtains by solving the Gorkov equation for the full Green's function in the pairing channel becomes larger than the width of the pairing susceptibility. In this situation, the solution of the Gorkov's equation has almost the same form as in the actual superconducting state where the pairing susceptibility has a $\delta$-functional form, i.e., system develops precursors to the $d$-wave superconducting state without developing a true superconductivity. It is essential, however, that as $G^{-1}(\mathbf{k}, \Omega) \propto e^{i \pi / 4} \sqrt{\Omega}$, the frequency shift due to $d$-wave precursors yields a pole in the full $G$ along an imaginary rather than a real frequency axis. This in turn yields the spectral function in the form

$$
A_{\beta=0}(\Omega) \propto \sqrt{\Omega} \frac{\Omega+\Omega_{0}}{\Omega^{2}+\Omega_{0}^{2}}
$$

where explicit computations yielded [5] $\Omega_{0}=\Omega_{0}(k) \approx$ $0.68 \omega_{\mathrm{sf}} \xi^{2}\left(\cos k_{x}-\cos k_{y}\right)^{2}$. This spectral function does not possess a sharp quasiparticle pole, but nevertheless it shows a "gap-like" behavior. Indeed, $A(\omega)$ rapidly increases at low frequencies, reaches half maximum at $\Omega \sim 0.2 \Omega_{0}$, then passes through a broad maximum at $\Omega=\Omega_{0}$, and slowly decreases at higher frequencies. This behavior, shown in Fig. 1, is in a good agreement with the form of the photoemission curve observed in experiments below $T^{*}$ [2].

We now study how the spectral function changes as one takes thermal effects into consideration. We assume that the conditions for $d$-wave precursors are satisfied (i.e., the width of the pairing susceptibility is smaller than the frequency shift obtained by solving the Gorkov's equation), but the self-energy, and, hence inverse bare 


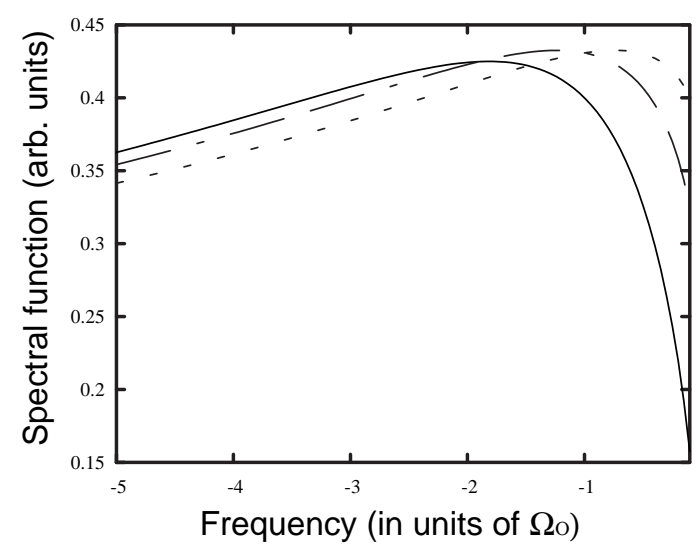

FIG. 1. The plot of the spectral function $A(\Omega)$ given by Eq. (6). Solid, dashed-dotted and dotted lines correspond to $\bar{\beta}=0,1 / 2$ and 1 , respectively. The spectral function for $\bar{\beta}=0$ displays the LEG and the broad maximum at higher frequencies. As $\bar{\beta}$ increases with temperature, the LEG is progressively filled in by excitations.

Green's function for the pairing channel, has the form of Eq. (4) with $\beta>0$. For the spectral function, we then obtain instead of (5)

$$
A(\Omega) \propto \frac{\alpha(\Omega) \sqrt{\Omega}\left(\Omega \frac{1+\alpha^{2}(\Omega)}{2}+\Omega_{0}\right)}{\left(\Omega \frac{1+\alpha^{2}(\Omega)}{2}\right)^{2}+\Omega_{0}^{2}-\Omega \Omega_{0}\left(1-\alpha^{2}(\Omega)\right)} .
$$

Here it is convenient to rewrite $\alpha(\Omega)$ as $\alpha(\Omega)=1+$ $\bar{\beta} \sqrt{\Omega_{0} / \Omega}$ where $\bar{\beta}=\beta /\left(0.82 \xi\left|\cos k_{x}-\cos _{y}\right|\right)$. The plots of $A(\Omega)$ for various values of $\bar{\beta}$ are presented in Fig. 11. We see that the LEG is progressively filled in as $\bar{\beta}$ increases from 0.5 to 1 . This happens chiefly because thermal fluctuations eliminate the $\sqrt{\Omega}$ dependence at small frequencies. Clearly, the temperature where the LEG disappears depends on $\mathbf{k}$ being the largest near the points where the $d$-wave gap has the largest value. The high-frequency part of $A(\Omega)$, which possesses a broad maximum, is a bit more robust and disappears at somewhat larger $\bar{\beta}$. In other words, as temperature increases, thermal excitations first fill the LEG, and then, at somewhat higher $T$, destroy the broad maximum at $\Omega_{0}$. This behavior seems to be consistent with both photoemission [3] and tunneling experiments 断. Notice that this mechanism is different from a conventional thermal smearing of the gap which in our approach would would correspond to a thermal broadening of the pairing susceptibility, $\chi^{s c}(\Omega)$. We now need to find the actual temperature dependence of $\beta$ and obtain the onset temperature for the LEG which can be directly compared with the data. For this, we compute the self-energy numerically by performing the summation over $n$ in (2) without simplifying the form of the integrand, i.e., without assuming that $\Omega_{m} \ll v \xi^{-1}$. We performed computations for $T=\omega_{\mathrm{sf}} / 2, T=\omega_{\mathrm{sf}}$ and $T=2 \omega_{\mathrm{sf}}$ and in all three cases found that the self-energy

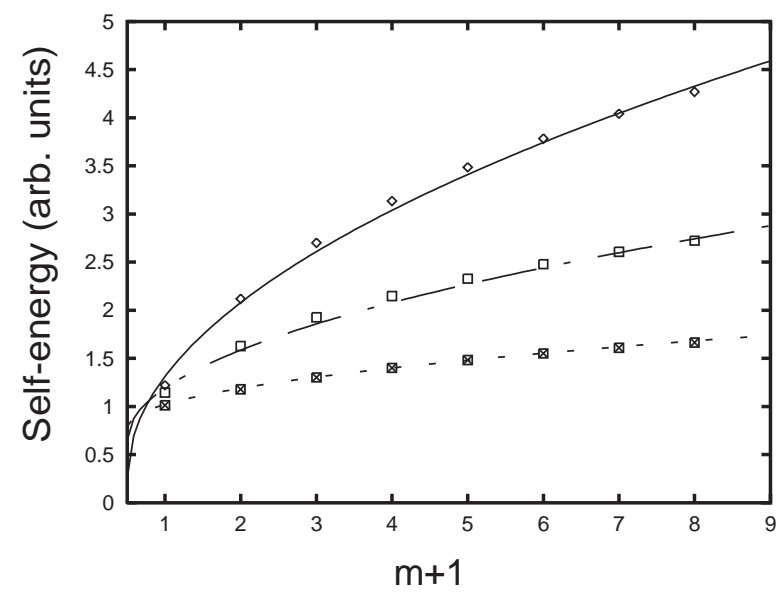

FIG. 2. The results for the full self-energy (2) for first 8 Matsubara frequencies $\Omega_{m}=\pi T(2 m+1)$ and the fits to $\sqrt{\Omega_{m}}+\beta \sqrt{\omega_{s f} / 2}$ dependence. Solid, dashed-dotted and dashed lines correspond to $T=\omega_{\mathrm{sf}} / 2, T=\omega_{\mathrm{sf}}$ and $T=2 \omega_{\mathrm{sf}}$, respectively.

for external fermionic frequencies $\Omega_{m}=\pi T(2 m+1)$ and $0 \leq m \leq 7$ can be well approximated by the same form as in (凷), i.e. $\Sigma\left(\mathbf{k}_{\mathrm{hs}}, \Omega_{m}\right) \propto\left(\sqrt{\Omega_{m}}+\beta \sqrt{\omega_{\mathrm{sf}} / 2}\right.$. The accuracy of the fit is demonstrated in Fig. 2. We emphasize that this procedure shows that the exact self-energy follows the $\sqrt{\Omega}$ dependence in a much larger frequency range than one may expect from the approximate analytical treatment. The overall factor in the fit decreases a bit with increasing temperature. This last renormalization, however, can be absorbed into the renormalization of $g$ and is irrelevant as the positions of both the LEG and the broad maximum in $A(\Omega)$ are governed by $\Omega_{0}$, which does not depend on the coupling strength as long as $g>g_{0}$.

We now present the numbers. We obtained $\beta=$ $0.24(0.38), 1.69(2.57)$ and $6.82(10.63)$ for $v \xi^{-1} / \Delta=1(2)$ and $T / \omega_{\mathrm{sf}}=0.5,1$ and 2 , respectively. We see that $\beta$ rapidly increases with increasing temperature. The increase roughly follows $T^{\nu}$ dependence with $\nu$ somewhat larger than 2, i.e., it is much stronger than the linear in $T$ increase which one would expect for a purely classical self-energy. On the other hand, already for $T=\omega_{\text {sf }} / 2$, the value of $\beta$ is about $40 \%$ smaller than in the Fermiliquid theory. This collaborates our earlier observation in (3) that the Fermi-liquid behavior is altered already at low $T$, but there is a wide intermediate temperature range where the thermal part of the self-energy interpolates between quadratic and linear in $T$ behavior.

Using $\bar{\beta}=0.5$ as a criteria for the onset of the LEG, we find that the LEG near $(0, \pi)$ is filled in at $T \sim T^{*}=0.6 \omega_{\mathrm{s}} \xi^{1 / \nu}$. A fit to NMR shows that $T^{*} \sim 150-200 K$. Away from $(0, \pi)$, the LEG is filled in at smaller temperatures which roughly follows $T^{*}(\mathbf{k})=T^{*}\left(\left|\cos k_{x}-\cos k_{y}\right| / 2\right)^{1 / \nu}$. Both of these re- 
sults are in quantitative agreement agreement with the data [2,3]. In addition, as one moves towards the zone diagonal, i.e., goes away from the hot spots, the overall strength of the $\sqrt{\Omega}$ term in the self-energy decreases. This pushes the system back towards a weak coupling limit and causes an additional decrease in $T^{*}(\mathbf{k})$. Simultaneously, one should gradually recover some features of a conventional quasiparticle peak in $A(\Omega)$. This last effect has also been observed in recent experiments [3]. Finally, a fit to the NMR data shows that the ratio $T / \omega_{\text {sf }}$ flattens at $\sim 1.4-1.5$ slightly above $T^{*}$ and remains constant upto much higher $T$. Then, $\bar{\beta}$ also flattens at a value $\sim 1$. This $\bar{\beta}$ still yields a broad maximum in $A(\Omega)$ above $T^{*}$, in agreement with the data.

We also emphasize that in our approach, the LEG gradually appears at $T \sim T^{*}(\mathbf{k})$ without a break up of the Fermi surface. In other words, the present theory predicts that above $T_{\mathrm{c}}$, there still exists a quasiparticle peak at a vanishing frequency at $\mathbf{k}=\mathbf{k}_{\mathrm{F}}$, The residue of the peak, however, decreases as one moves away from the zone diagonal. Near $(\pi, 0)$ it scales as $\left(g_{0} / g\right)^{2}$ which seems too small to be detected by current photoemission experiments. Notice in this regard that photoemission data show that the quasiparticle peak is clearly present near the zone diagonal, and gradually disappears as one approaches $(0, \pi)$. The tunneling experiments 顿, which measure the momentum averaged spectral density, also seem to observe a coherent peak above $T_{c}$ in the unoccupied part of the density of states.

A final note. In the present consideration, the LEG and the broad maximum in $A(\omega)$ at a finite frequency are both $d$-wave precursors in a Fermi liquid with strong magnetic fluctuations. A number of other scenarios for a pseudogap have also been proposed [6, [, 9, 10]. In particular, several groups argued [6, 99,7] that the broad maximum in the spectral function is a precursor of an antiferromagnetic SDW state. In the present calculations, SDW precursors do not appear because the fermionic self-energy is an increasing function of frequency upto very large frequencies. The SDW precursors, on the contrary, can appear only if the self-energy decreases with frequency [6]. We found, however, that the form of the spectral function at $\Omega \geq v \xi^{-1}$ is highly sensitive to the high-frequency form of the spin susceptibility. Namely, we did calculations using $\chi(\mathbf{q}, \Omega)$ in which the $\omega^{2}$ term is substituted by a hard cutoff at $\omega \sim \Delta$ and found that the classical behavior sets up at much smaller temperatures. In this last case, for $T>T^{*}$, the dominant contribution to (2) comes from the $n=0$ term, i.e., spin fluctuations are essentially static. These fluctuations yield the fermionic self-energy which decreases with frequency and gives rise to SDW precursors at $\Omega \geq v \xi^{-1}$ provided that $\left(g / g_{0}\right)^{2}\left(T / v \xi^{-1}\right) \geq 1 / 4$ which is satisfied above $T^{*}[7]$. Quantum SDW precursors can also appear at high frequencies provided that feedback effects substantially reduce the spin damping [5,6,9. In view of this nonuni- versality, it is possible that the broad maximum in $A(\omega)$ at high frequencies is actually a mixture of $d$-wave and SDW precursors.

To summarize, in this paper we discussed the thermal variation of the fermionic spectral function $A(\Omega)$ in the spin-fermion model for underdoped cuprates. We found that in the strong coupling limit, the fermionic Green's function near $(0, \pi)$ behaves as $G^{-1}(\Omega) \propto(\sqrt{\Omega}+$ $\left.\beta \sqrt{\omega_{s f} / 2}\right)$ in the whole frequency range relevant to photoemission experiments. The frequency-independent term is due to thermal spin fluctuations. At low temperatures, the $\sqrt{\Omega}$ term dominates and yields the LEG in $A(\Omega)$ and a broad maximum at larger frequencies. As $T$ increases, spin fluctuations progressively acquire a classical, quasistatic behavior. This effect yields a rapid increase of $\beta$ with temperature which in turn gives rise to a destruction of the LEG. We found that the LEG disappears at $T=T^{*} \sim 0.6 \omega_{\mathrm{sf}} \xi^{1 / \nu}$ which is $150-200 K$ near $(0, \pi)$ and decreases as one moves towards zone diagonal.

It is our pleasure to thank J.C. Campuzano, L. Gorkov, M. Norman, M. Onellion, D. Pines, J. R. Schrieffer and B. Stojković for useful conversations. The research was supported by NSF DMR-9629839 (A.C) and the STCS through NSF-grant DMR91-20000 as well as the Deutsche Forschungsgemeinschaft (J.S.). Part of this work has been done at the Aspen Center for Physics.

[1] see e.g, D.J. Scalapino, Phys. Rep. 250, 329 (1995); A.V. Chubukov, D. Pines, and B.P. Stojkovic, J. Phys. Condens. Matter 8, 10017 (1996) and references therein.

[2] D.S. Marshall et al., Phys. Rev. Lett. 76, 4841 (1996); H. Ding et al., Nature 382, 51 (1996); Z.-X. Shen and J.R. Schrieffer, Phys. Rev. Lett. 78, 1771 (1997)

[3] M.R. Norman et al., cond-mat/9710163.

[4] Ch. Renner et al., Phys. Rev. Lett., submitted.

[5] A.V. Chubukov, cond-mat/9709221. For the earlier studies of the fermionic self-energy see A.J. Millis, Phys. Rev. B 45, 13047 (1992); A. Chubukov, ibid 52, R3840 (1995); B. L. Altshuler, L.B. Ioffe and A. J. Millis, ibid 52, 415, 5563 (1995).

[6] A.V. Chubukov and D.K. Morr, Phys. Rep., 288, 355 (1997) and references therein; A.V. Chubukov, D.K. Morr and K. Shakhnovich, Phil. Mag. B 74, 563 (1996).

[7] J. Schmalian, D. Pines and B.P. Stojkovic, Phys. Rev. Lett., submitted.

[8] V. Barzykin and D. Pines, Phys. Rev. B, 52, 13585 (1995).

[9] A.P. Kampf and J.R. Schrieffer, Phys. Rev. B 42, 7967 (1990); E. Dagotto, A. Nazarenko and A. Moreo, Phys. Rev. Lett 74, 310 (1995).

[10] X.G. Wen and P.A. Lee, Phys. Rev. Lett. 76, 503 (1996); R. Laughlin, ibid 79, 1726 (1997); V. Emery and S. Kivelson, Nature 374, 434 (1995). 